\title{
Exploratory Analysis of Acoustic Emissions in Steel using Dictionary Learning
}

\author{
Sergio Martin-del-Campo ${ }^{1}$, Fredrik Sandin ${ }^{2}$, Stephan Schnabel ${ }^{3}$, Pär Marklund ${ }^{4}$, Jerker Delsing ${ }^{5}$
}

\begin{abstract}
Analysis of acoustic emissions (AE) from steel deformation is a challenging condition monitoring problem due to the high frequencies and data rates involved, and the difficulty to separate signals from noise. The problem to characterize and identify different $\mathrm{AE}$ sources calls for methods that goes beyond conventional time and frequency domain analysis. Feature learning is common in the field of machine learning and is successfully used to approximate and classify other kinds of complex signals. Former studies show that $\mathrm{AE}$ classification results depend on the choice of predefined features that are extracted from the raw AE signal, but little is known about feature learning in this context. Here we use dictionary learning and sparse coding to optimize a set of shift-invariant features to the AE signal measured in a steel tensile strength test. The specimen undergoes elastic and plastic deformation and eventually cracks. We investigate the learned features and their repetition rates and use principal component analysis (PCA) to illustrate that the resulting sparse AE code is useful for classification of the three strain stages, without reference to the signal amplitude. Therefore, feature learning is a potentially useful approach to the $\mathrm{AE}$ analysis problem, which also opens up for further studies of automated methods for anomaly detection in AE.
\end{abstract}

\section{INTRODUCTION}

Acoustic emissions (AE) are transient elastic waves generated by the rapid release of energy in solid materials. For example, damage within or at the surface of steel in machine elements results in high-frequency $\mathrm{AE}$ [1]. AE is observed when steel undergo plastic deformation and when cracks are formed, such as in a tensile strength test where high force is applied to a specimen so that it ultimately ruptures, see Figure 1. Deformation processes in the specimen generate AE that can be measured using standard sensors.

Analysis of AE can provide early warning signals and information about machine component wear and maintenance needs. However, the data acquisition and analysis problem is challenging due to the high frequencies, data rates and noise levels involved. Traditionally, analysis of AE is based on handcrafted signal approximations and extraction of some particular features of the signal. For example, properties of the time domain signal like the AE amplitude, energy,

${ }^{1} \mathrm{~S}$. Martin-del-Campo is a Ph.D. student at the SKF-LTU University Technology Center and EISLAB, Luleå University of Technology (LTU), 97187, Luleå, Sweden; sergio. martindel campo@ltu.se

${ }^{2} \mathrm{~F}$. Sandin is with EISLAB, Luleå University of Technology (LTU), 97187, Luleå, Sweden; fredrik. sandineltu. se

${ }^{3} \mathrm{~S}$. Schnabel is a Ph.D. student at the SKF-LTU University Technology Center and the Div. of Machine Elements, Luleå University of Technology (LTU), 97187, Luleå, Sweden; stephan. schnabel@ltu.se

${ }^{4} \mathrm{P}$. Marklund is with the Div. of Machine Elements, Luleå University of Technology (LTU), 97187, Luleå, Sweden; par.marklund@ ltu.se

${ }^{5} \mathrm{~J}$. Delsing is with EISLAB, Luleå University of Technology (LTU), 97187, Luleå, Sweden; jerker. delsing@ltu.se
RMS or threshold-based event counts can be analyzed [2]. Perhaps the most common method is the high-frequency resonance technique, also known as the envelope method [3]. By extracting the envelope of the signal the sampling rate can be significantly reduced and conventional frequency domain analysis methods can be applied. However, using such rough approximations information is also lost and it has proven difficult to derive reliable models for detailed analysis of $\mathrm{AE}$ in condition monitoring applications. Can complementary information be extracted from AE using unsupervised machine learning methods like feature learning? For example, can feature learning methods be used to classify different strain stages in a tensile test specimen?

Former work in this area has focused on vibration signals and machine learning methods enabling for example approximation of the system-response and classification of faults [4], [5], [6]. Furthermore, there is evidence that matching pursuit is useful for classification of $\mathrm{AE}$ in concrete structures [7]. Approaches like these can also be useful for studies and processing of AE signals in steel. The increasing computational capabilities and number of mechanical systems that can be monitored thanks to modern measurement and Internet-ofThings communication technology motivates investigations of machine learning methods for automated fault detection and classification.

In this paper we apply a feature learning method to AE measured on a tensile test specimen. We present exploratory data analysis results obtained with a state-of-the-art dictionary learning method based on local orthogonal matching pursuit. The signal model is based on general assumptions like linearity and sparsity in a transformed space. We study changes in the AE signal when the test specimen is sequentially exposed to elastic and plastic deformation with resulting crack formation and rupture. Inspection of the time domain signal reveal changes in the signal amplitude between the elastic, plastic and cracking strain stages, but that analysis is not easily automated. Instead, we use a model where the signal is represented as a linear superposition of noise and shift-invariant waveforms with compact support, which are optimized to the signal using dictionary learning. The dictionary of waveforms (called atoms) is learned in an unsupervised way.

We observe clear differences in the signal approximations corresponding to the three strain stages, without reference to the signal amplitude. These results indicate that dictionary learning is a useful complementary approach to analyse AE and identify morphological features encoding information about the AE sources and the system transfer function. 


\section{DiCTIONARY LEARNING METHOD}

The dictionary learning method is based on the model developed by Smith and Lewicki [8], and it is inspired by earlier work of Olshausen and Field [9]. Smith and Lewicki discovered that atoms learned from speech closely resemble cochlear impulse response functions (revcor filters), which indicates that speech has adapted to the ear [8]. Our working hypothesis here is that features that characterize other signals like AE can be learned in a similar manner. The method decomposes a signal, $x(t)$, in a linear superposition of noise and atomic waveforms with compact support

$$
x(t)=\sum_{m=1}^{M} \sum_{i=1}^{N_{m}} a_{m, i} \phi_{m}\left(t-\tau_{m, i}\right)+\epsilon(t) .
$$

The functions $\phi_{m}(t)$ are atoms that represent morphological features of the signal and $M$ indicates the total number of such atoms. The variable $N_{m}$ refers to the number of instances of atom $\phi_{m}$, and the temporal position and amplitude of the $i$-th instance of atom $\phi_{m}$ are denoted by $\tau_{m, i}$ and $a_{m, i}$, respectively. The set of $M$ atoms defines a dictionary

$$
\Phi=\left\{\phi_{1}, \cdots, \phi_{M}\right\}
$$

The values of $\tau_{i}$ and $a_{i}$ are determined with an efficient orthogonal matching pursuit (OMP) algorithm [10], [11].

The OMP algorithm is an iterative process that decomposes the signal in the dictionary of atoms. The algorithm operates on the residual of the signal, which initially is the measured signal to be decomposed. The algorithm calculates the cross-correlation between the residual and all the elements of $\Phi$. The atom with the maximum cross correlation (inner product) for all possible timeshifts is selected and it defines an atom-occurrence event. The time of the event is defined as $\tau_{i}$ and the inner product as $a_{i}$. This process is repeated until a stopping criterion is fulfilled, which for example can be defined in terms of the sparsity level or a minimum signal to residual ratio. In each iteration, the residual is updated by subtracting the event, $a_{i} \phi_{m(i)}\left(t-\tau_{i}\right)$, from the residual. In addition, all coefficients $a_{i}$ are updated with an orthogonal projection of the signal onto the set of selected atoms. This way, OMP results in a lower approximation error compared to ordinary matching pursuit at the expense of higher computational cost. For further details about the lowcomplexity implementation of OMP used here see [12].

The problem to learn the dictionary, $\Phi$, of atoms is the main challenge. The goal is to automatically optimize the set of atomic waveforms $\phi_{m}$ in the dictionary, $\Phi$, to a particular signal domain like AE. One solution to this problem can be obtained by rewriting Eq. (1) in probabilistic form

$$
p(x \mid \Phi)=\int p(x \mid a, \Phi) p(a) d a \approx p(x \mid \hat{a}, \Phi) p(\hat{a}),
$$

where $\hat{a}$ is the maximum a posteriori (MAP) estimate of $a$ [13],

$$
\hat{a}=\arg \max _{a} p(a \mid x, \Phi)=\arg \max _{a} p(x \mid a, \Phi) p(a),
$$

that is generated by the orthogonal matching pursuit [8]. The prior of the amplitude, $p(a)$, is defined to promote sparse coding in terms of statistically independent atoms [9]. We assume that the likelihood, $p(x \mid a, \Phi)$, is Gaussian. This results in a learning algorithm that involves gradient ascent on the approximate log data probability defined by

$$
\frac{\partial}{\partial \phi_{m}} \log (p(x \mid \Phi))=\frac{1}{\sigma_{\epsilon}^{2}} \sum_{i} a_{m, i}[x-\hat{x}]_{\tau_{m, i}} .
$$

The gradient of each atom in the dictionary is proportional to the sum of residuals within the support of each atom event.

In order to use the gradient for dictionary learning we introduce a stepsize parameter, $\eta$, which controls the dictionary learning rate. The resulting update rule for atoms is

$$
\phi_{m} \rightarrow \phi_{m}+\frac{\eta}{\sigma_{\epsilon}^{2}} \sum_{i} a_{m, i}[x-\hat{x}]_{\tau_{m, i}} .
$$

This means that the learning rate depends on the activation rate of atoms, which implies that the learning rate of atoms can be different and that some atoms may not learn at all.

\section{EXPERIMENT}

We are interested in the characteristics of the dictionarybased representation and residual of $\mathrm{AE}$ from steel when the stress-strain state changes. Therefore, we consider a standardized ferrite sheet metal specimen subject to a tensile test. The selection of ferrite steel is motivated by the single phase structure, which reduces the complexity. Figure 1 displays the geometry and dimensions of these samples as well as the position of the AE sensor. The sensor is glued with bees wax, which serves as an impedance matching interface.
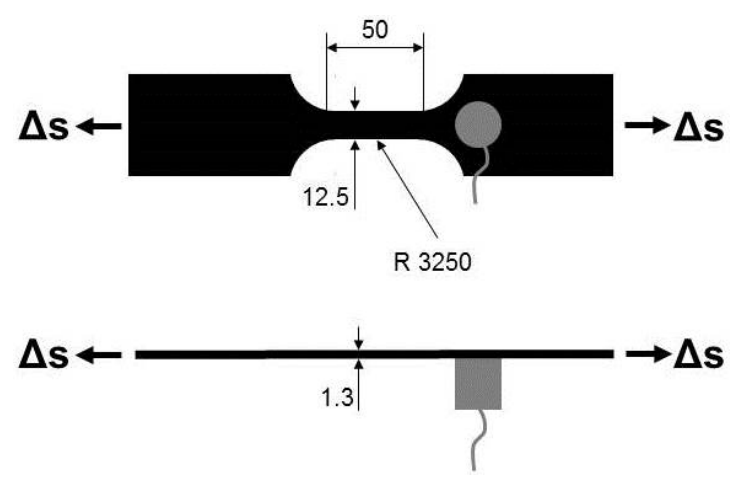

Fig. 1: Schematic drawing of the ferrite tensile test specimen, including the sensor position. Dimensions are in $\mathrm{mm}$.

The tensile test was executed with a hydraulic test frame for tension and compression (Dartec) in combination with a digital control unit (Instron). Samples were drawn apart with a constant displacement speed of $\Delta s=0.1 \mathrm{~mm} / \mathrm{s}$. A TTL trigger signal was used to synchronize tensional force and acoustic emission measurement recordings. Acoustic emissions were measured with an accelerometer (WS $\alpha$ ) in combination with a preamplifier (Physical Acoustic 2/4/6C) that included a band pass filter in the $100 \mathrm{kHz}$ to $1.2 \mathrm{MHz}$ range. The signals were collected with a 14-bit PCI digitizer 
(GaGe OSC-432-007) at a sample rate of $5 \mathrm{MS} / \mathrm{s}$. At this sample rate, the on-board memory allowed for a maximum recording time of $132 \mathrm{~s}$, which is sufficient for the tests.

We apply dictionary learning to the recorded AE data, which encodes information about the steel as it transforms from the elastic strain stage to the plastic and crack propagation stages. The AE data is processed with our Matlab/C++ implementations of local OMP [12] for creating the sparse representation and Smith and Lewicki's algorithm [8] for dictionary learning.

The signal is divided in consecutive $10 \mathrm{~ms}$ segments with $50 \mathrm{k}$ samples each. The segments range from when force is first applied until rupture of the specimen after 4310 segments. Segments are preprocessed to have zero mean and unit variance. The OMP is stopped at $90 \%$ sparsity, which means that $50 \mathrm{k}$ AE samples are modeled with $5 \mathrm{k}$ atom events (compression ratio of 0.1 ). We use a step size of $\eta=1 \times 10^{-6}$ for dictionary update. Initially the dictionary contains eight atoms of length fifty sampled from a Gaussian distribution with zero mean. The dictionary is trained with $1 \mathrm{k}$ randomly selected segments corresponding to the initial elastic deformation of the specimen. Random selection is used to compensate for the relatively short duration of elastic deformation data (210 segments). The resulting dictionary is further propagated by training with consecutive segments corresponding to plastic deformation followed by crack formation. Atoms can grow in length and are normalized after each update.

\section{RESUlTS}

Our aim is to investigate how the learned atoms and related AE model parameters change when the steel specimen is subject to strain during the tensile test.

An initial dictionary learning experiment is carried out with the entire 46.2 seconds of $5 \mathrm{MS} / \mathrm{s} \mathrm{AE}$ data recorded in the experiment, see Section III for details. Preliminary assessment of the results reveals that the atoms do not converge in the elastic domain due to the relatively short duration of the elastic signal of about two seconds. Therefore, training with elastic $\mathrm{AE}$ data is performed with $1 \mathrm{k}$ randomly selected segments so that 10 seconds of elastic data is processed. Thereafter the resulting dictionary is trained (with dictionary propagation) using the remaining 44 seconds of AE data, which corresponds to plastic deformation and crack propagation.

The resulting dictionaries for the elastic, plastic and crack propagation cases are shown in Figure 2, each including eight atoms. The elastic dictionary is obtained after training on 1000 segments, which corresponds to 10 seconds of AE. The plastic dictionary is extracted after about 20 seconds of training, which is halfway through the plastic deformation. The crack propagation dictionary is obtained after 43 seconds of training, immediately before rupture of the specimen. The learned atoms are normalized and the eight panels have the same scale along the $y$-axis. Each panel show the atoms for the elastic case (top), plastic case (middle) and crack propagation case (bottom). The panels illustrate how each

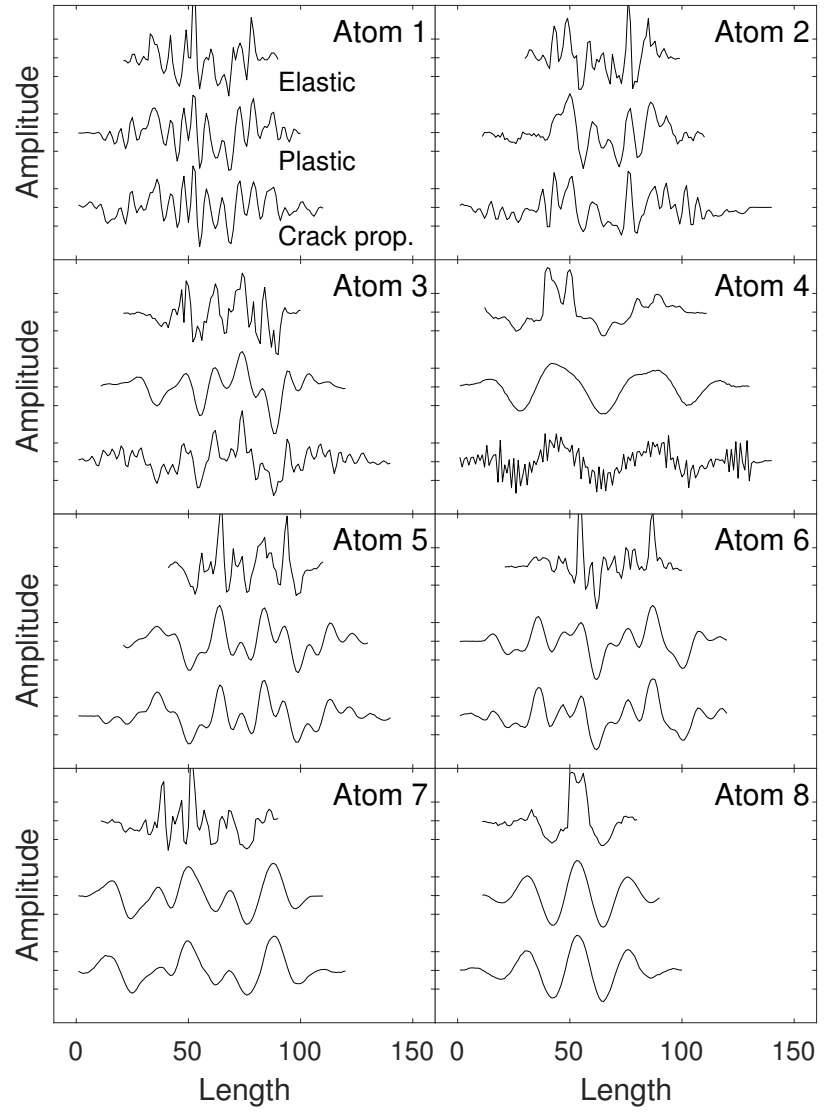

Fig. 2: Dictionary of atoms learned from AE recorded from the steel specimen under strain (elastic, plastic and crack).

atom evolve during the training process at different strain stages. Atoms are ordered by decreasing atom repetition rates in the crack propagation case.

Visual inspection of the atoms reveal some differences between the atoms learned in the three different strain stages. For example, atom 4 is more noise-like in the crack propagation case but the shape is otherwise similar to that in the plastic case. Atom 6 have similar shapes and lengths in the plastic and crack cases. Other atoms are longer in the crack case than in the plastic case, but the extended tails have low RMS amplitude and are natural results of long duration training. Atoms learned in the elastic case are more noise-like compared to the plastic and crack cases, which is a consequence of a relatively low signal amplitude and SNR in that case. However, atoms 1, 4 and 8 appear somewhat similar in the elastic, plastic and crack cases.

In Figure $3 \mathrm{a}$ we present the average repetition rates of each atom over 50 segments ( 0.5 seconds of AE data). Error bars denote the corresponding standard deviations. In the elastic case the atom repetition rates are rather similar for different atoms, which may be a consequence of the low amplitude and SNR in that case. When the test specimen enters the plastic deformation stage the atoms quickly adapt and the repetition rates varies significantly between atoms. Different repetition rates are observed also in the case of crack propagation. The accuracy of the sparse approximation 

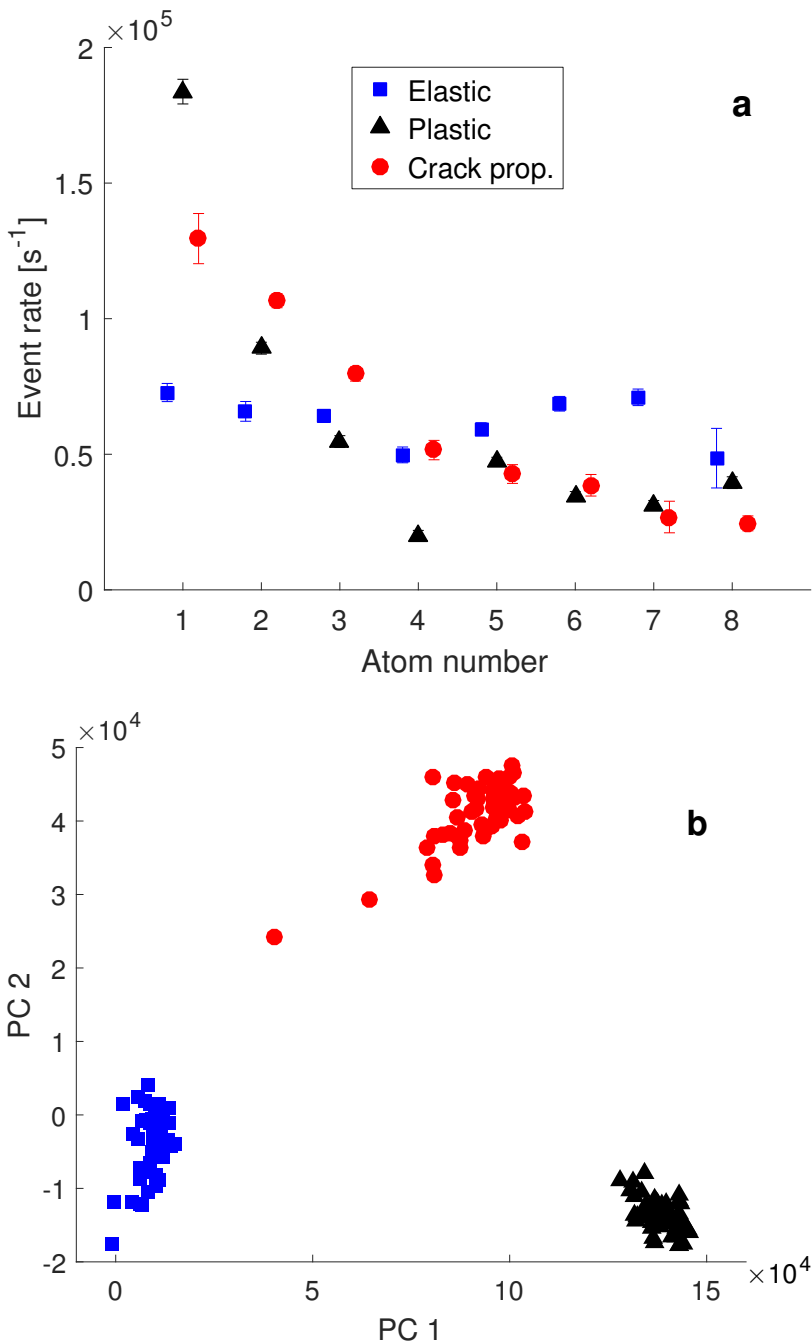

Fig. 3: Clustering of event rates with PCA. a) Number of atom events per second versus atom number. b) Event rates projected on the first two principal components.

differs as well. The maximum fidelity obtained in the elastic case is $6.5 \mathrm{~dB}$, while in the plastic case $16 \mathrm{~dB}$ is obtained. In the crack propagation case the fidelity oscillates between $2.4 \mathrm{~dB}$ and $32 \mathrm{~dB}$.

Next we perform principal component analysis (PCA) on the atom repetition rates for the $3 \times 50 \mathrm{AE}$ data segments used to prepare Figure $3 \mathrm{a}$. Figure $3 \mathrm{~b}$ shows the projection of the repetition rates on the first and second principal components, which defines the $2 \mathrm{D}$ plane where the data have maximum variance. Three well-separated clusters are formed, which corresponds to the three different strain stages. The atom repetition rates depend on the morphological structure of the signal, not on the scale of the signal amplitude. These results indicate that even though the learned atoms are similar in the plastic and crack cases, the sparse signal approximations are different in the three strain stages.

\section{Discussion}

We study AE measured during a ferrite tensile test using an unsupervised dictionary learning method based on orthogo- nal matching pursuit and probabilistic gradient ascent. Using PCA we illustrate that the atom repetition rates in the AE signal approximation are different in the elastic, plastic and crack propagation strain stages of the test specimen. This makes strain classification straightforward in the experiment considered here, without reference to the signal magnitude which is key in conventional time domain analysis. It is expected that the system response of the test specimen should change due to deformation and crack formation. Some learned atoms have different shapes in the three strain stages. Further work is required to investigate the significance of these changes, and whether the transitions between strain stages can be detected. The latter is challenging because it takes some time for the learned dictionary of atoms to adapt under changing conditions. This implies that the immediate transient effect of plastic deformation and crack formation is expected in the model residual, $\epsilon(t)$. These results motivate further investigations of feature learning for AE classification and anomaly detection, for example in condition monitoring applications.

\section{ACKNOWLEDGMENT}

This work is partially supported by SKF via their University Technology Center at LTU, and the Kempe Foundations.

\section{REFERENCES}

[1] D. Mba and R. B. K. N. Rao, "Development of acoustic emission technology for condition monitoring and diagnosis of rotating machines: Bearings, pumps, gearboxes, engines, and rotating structures," The Shock and Vibration Digest, vol. 38, no. 1, pp. 3-16, 2006.

[2] A. Morhain and D. Mba, "Bearing defect diagnosis and acoustic emission," in Proceedings of the Institution of Mechanical Engineers, Part J: Journal of Engineering Tribology, 2003, pp. 217-257.

[3] P. McFadden and J. Smith, "Vibration monitoring of rolling element bearings by the high-frequency resonance technique - a review," Tribology International, vol. 17, no. 1, pp. 3-10, 1984.

[4] K. Worden, W. J. Staszewski, and J. J. Hensman, "Natural computing for mechanical systems research: A tutorial overview," Mechanical Systems and Signal Processing, vol. 25, no. 1, pp. 4 - 111, 2011.

[5] S. Martin del Campo, K. Albertsson, J. Nilsson, J. Eliasson, and F. Sandin, "FPGA prototype of machine learning analog-to-feature converter for event-based succint representation of signals," in IEEE Int Workshop Mach Learn Signal Process, Southampton, UK, 2013.

[6] S. Martin-del Campo and F. Sandin, "Towards zero-configuration condition monitoring based on dictionary learning," in Signal Processing Conference (EUSIPCO), 23rd European, Aug 2015, pp. 1306-1310.

[7] S. P. Ebenezer, A. Papandreou-Suppappola, and S. B. Suppappola, "Classification of acoustic emissions using modified matching pursuit," Journal on Applied Signal Processing, vol. 3, pp. 347-357, 2004.

[8] E. Smith and M. S. Lewicki, "Efficient auditory coding," Nature, vol. 439, no. 7079, pp. 978-982, 2006.

[9] B. A. Olshausen and D. J. Field, "Sparse coding with an overcomplete basis set: A strategy employed by v1?" Vision Research, vol. 37, pp. 3311-3325, 1997.

[10] S. Mallat and Z. Zhang, "Matching pursuits with time-frequency dictionaries," IEEE T Signal Proces, 1993.

[11] Y. C. Pati, R. Rezahfar, and P. S. Krishnaprasad, "Orthogonal matching pursuit: Recursive function approximation with applications to wavelet decomposition," in Signals, Systems and Computers. 1993 Conference Record of The Twenty-Seventh Asilomar Conference on, vol. 1, November 1993, pp. 40-44.

[12] B. Mailhe, R. Gribonval, F. Bimbot, and P. Vandergheynst, "A low complexity orthogonal matching pursuit for sparse signal approximation with shift-invariant dictionaries," in Proc IEEE Int Conf Acoust Speech Signal Process, ICASSP 2009., April 2009, pp. 3445-3448.

[13] E. Smith and M. S. Lewicki, "Efficient coding of time-relative structure using spikes," Neural Computation, no. 1, pp. 19-45. 